\title{
On the relation between photospheric magnetic field and chromospheric emission in the quiet Sun
}

\author{
Maria A. Loukitcheva ${ }^{1,2}$, Sami K. Solanki ${ }^{2}$ \\ and Stephen M. White ${ }^{3}$ \\ ${ }^{1}$ Astronomical Institute, St. Petersburg University, 198504 St. Petersburg, Russia \\ email: marija@astro.spbu.ru \\ ${ }^{2}$ Max-Planck-Institut für Sonnensystemforschung, D-37191 Katlenburg-Lindau, Germany \\ ${ }^{3}$ Astronomy Department, University of Maryland, College Park, MD 20742
}

\begin{abstract}
In this contribution we present an observational study of the interaction of the photosphere with different chromospheric layers. We study the correlations between emissions at varying temperature from the temperature minimum region (UV continuum at $1600 \AA$ from TRACE) through the low chromosphere (CaII K-line from BBSO) to the middle chromosphere (continuum at $3.5 \mathrm{~mm}$ from BIMA) and photospheric magnetic field from MDI/SOHO. For the first time millimeter observational data are included in such analysis.

We report a high degree of correlation between considered emissions formed at different heights in the chromosphere. A power law is found to be a good representation for the relationship between photospheric magnetic field and chromospheric emissions at all considered wavelengths. Our analysis shows that the dependence of chromospheric intensities on magnetic field is different for the network and internetwork regions. In the network a power law provides the best fit with the exponent being close to $0.5-0.6$, while almost no dependence of chromospheric intensity on magnetic flux is found for the cell interiors. The obtained results support the idea of different heating mechanisms acting in the network (magnetic) and cell interiors (acoustic).
\end{abstract}

Keywords. Sun: magnetic fields - Sun: chromosphere - Sun: radio radiation - Sun: photosphere

\section{Introduction}

In the quiet-Sun chromosphere, even far from the activity complexes, magnetic field is believed to be responsible for chromospheric structuring, which displays a variety of inhomogeneities at different spatial scales. The primary quiet-Sun brightness patterns, revealed from chromospheric images, include chromospheric network, located at the boundaries of supergranular cells, and bright points in the interior of the cells - internetwork grains. For the primary chromospheric diagnostic, which is the emission in the cores of the calcium $\mathrm{K}$ and $\mathrm{H}$ resonance lines, a strong correlation between the presence and amount of the quiet-Sun magnetic flux and excess core emission is well established (e.g. Leighton 1959; Schrijver et al. 1989). And naturally these resonance lines commonly serve as indicators of changes in the chromospheric structure related to global magnetic activity and heating of the outer atmosphere of the Sun and cool stars (Rutten \& Uitenbroek 1991; Schrijver et al. 1989).

Apart from the chromospheric emission related to the magnetic field, Schrijver (1987, 1992) has suggested that a part of the radiative flux from solar and stellar chromospheres is of nonmagnetic origin, it is referred to as basal flux, and is believed to be due to acoustic heating of the chromosphere. Consequently, these two components - basal and strongfield, are the basic components, which determine the properties of the solar brightness 
Table 1. Overview of data taken on May 18, 2004

\begin{tabular}{ccccc}
\hline Instrument & $\lambda$ & $\Delta \lambda(\AA)$ & Spatial resolution $(\operatorname{arcsec})$ & Pixel size $(\operatorname{arcsec})$ \\
\hline MDI/SOHO & $6768 \AA$ & 0.094 & 4 & 1.96 \\
TRACE & $1600 \AA$ & 275 & 1 & 0.5 \\
BBSO & $3933 \AA$ & 0.6 & $2-4$ & 0.645 \\
BIMA & $3.5 \mathrm{~mm}$ & & 12 & 3.0 \\
\hline
\end{tabular}

structures. Thus the chromospheric network is known to display a one-to-one spatial correspondence with regions of enhanced photospheric magnetic field (Skumanich et al. 1975; Sivaraman et al. 1982; Nindos \& Zirin 1998), while the origin (magnetic or nonmagnetic) of the internetwork grains is a controversial issue.

Quantitative studies of the relationship between the chromosphere in calcium, both active and quiet, and magnetic field were carried out by a number of authors and naturally fall into two distinct classes. In a number of studies (see, e.g., Schrijver et al. 1989; Harvey \& White 1999) it was obtained that the relation between the K-line excess flux density and magnetic field flux density is best described by a power law with an exponent around $0.5-0.6$. This conclusion refers to different types of brightness structures, including active regions, decaying active regions, the enhanced network and quiet (weak) network. However, for quiet-Sun chromosphere Skumanich et al. (1975) and Nindos \& Zirin (1998) found no evidence of non-linear behaviour and obtained a linear correlation.

\section{Observational Data}

In this work to study the relation between the photosphere and different chromospheric layers we analyzed photospheric magnetograms from the Michelson Doppler Imager (MDI, Scherrer et al. 1995) on board of SOHO, UV images at $1600 \AA$ from the Transition Region and Coronal Explorer (TRACE, Handy et al. 1999), CaII K-line filtergrams from the Big Bear Solar Observatory (BBSO) and millimeter images at $3.5 \mathrm{~mm}$ from the Berkeley-Illinois-Maryland Array (BIMA), obtained on May 18, 2004. This is the first analysis that includes millimeter observational data. A quiet-sun region close to the disk center was observed for 3.5 hours. In Table 1 we give the overview of the data (including instruments, operating wavelengths, spatial resolution and image pixel size). We based our correlation analysis on the mean (averaged in time) images to avoid scatter introduced by chromospheric oscillations. Thus, by averaging the images we reduce significantly the scatter in the relationship and the noise in the magnetograms. In this work we studied images, averaged over the whole observational run of 3.5 hours and over halfhour time interval. The latter was used to check the stability of the derived structures. Prior to averaging careful spatial co-alignment of the observational data cubes was performed. Finally, the full disk MDI images were chosen as the coordinate reference against which all other mean images were co-aligned. The remaining image displacements do not exceed 1" and do not affect the results of the analysis. More details about the data and its reduction and alignment can be found in Loukitcheva et al. (2009).

\section{Results}

The resulted images of the solar chromosphere from the temperature minimum (TRACE $1600 \AA$ image) through the low chromosphere (CaII K-line filtergram) to the 


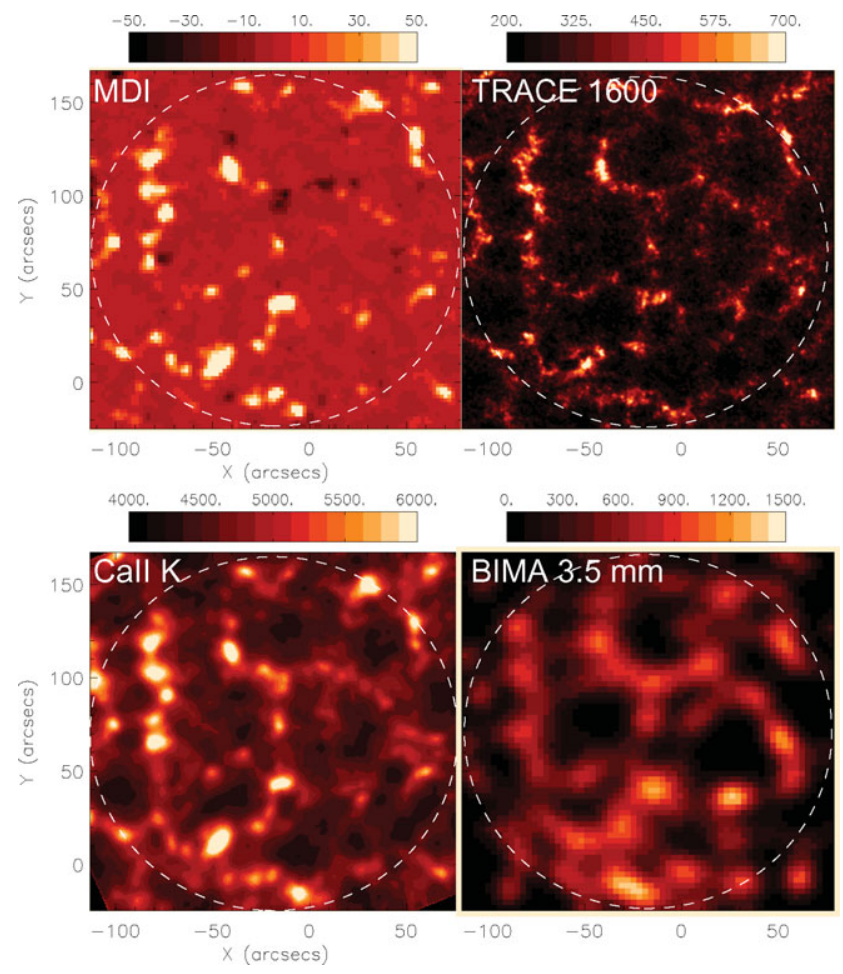

Figure 1. The solar chromosphere at 4 different wavelengths on May 18, 2004. From top left to bottom right: MDI longitudinal photospheric magnetogram, TRACE $1600 \AA$ image, CaII K-line center image from BBSO and BIMA image at $3.5 \mathrm{~mm}$. The images are created by averaging over $3.5 \mathrm{~h}$ observational run. Dashed circles mark the 96 arcsec BIMA FOV containing flux. $X$ and $Y$ axes are in $\operatorname{arcsec}$ from the disk center.

middle chromosphere (BIMA image at $3.5 \mathrm{~mm}$ ) at the original resolution of the instruments and the corresponding photospheric magnetogram can be seen in Fig. 1. All chromospheric maps and (unsigned) MDI magnetograms reveal clearly the same pattern, although at different spatial resolutions, most prominently on spatial scales of the enhanced magnetic flux and chromospheric network. The degree of spatial agreement between radio and other chromospheric radiation and photospheric magnetic field is more clearly visible in maps degraded to the BIMA resolution of $12 \operatorname{arcsec}$ (not shown).

Firstly, we computed pixel-to-pixel scatter plots for the pairs of images globally in the field of view and searched for the best linear and power-law fits (Fig. 2). By comparison of the least-square deviations of the best fits, we were able to determine confidently the non-linearity of the behaviour for all 3 considered chromospheric magnetic-intensity relationships. The exponents of the best power-law fits are listed in the last column of Table 2. The tightest relation is displayed by the calcium K-line, which is shown plotted in Fig. 2. The power law exponent of the relation, being 0.31, is smaller than those found by Schrijver et al. (1989) and Harvey \& White (1999) (see Sect. 1). This probably has to do with the smaller $B$ range available in the quiet Sun, although it cannot be ruled out that the calcium intensity does not increase so rapidly in the quiet Sun as in active regions. It is seen from Fig. 2 that the non-linearity of the relation is influenced mostly by weak chromospheric emission, that reveals a strong dependence on magnetic field. Thus, if we set a $B$ threshold of $20 \mathrm{G}$ we cannot distinguish between the quality of the linear and power-law fits for the relation between calcium intensity and magnetic field strength, as 


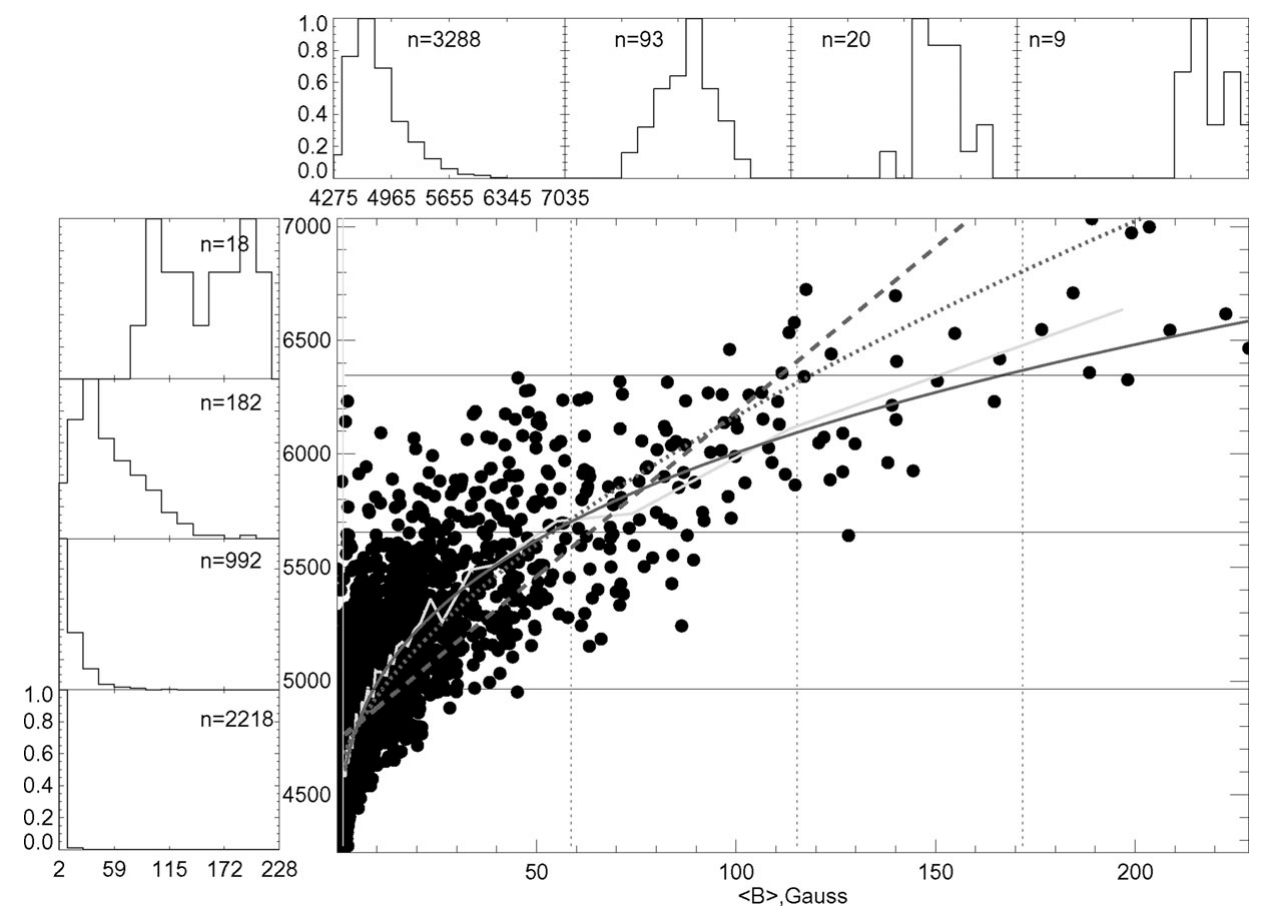

Figure 2. The Ca K-line intensity vs. the absolute value of the magnetic flux density for a 2 arcsec pixel size. The images are averaged in time over $3.5 \mathrm{~h}$. The vertical line at $1.5 \mathrm{G}$ represents the $1 \sigma$ noise limit of the magnetogram signal. The fit results are represented by a solid curve for a power-law fit with free exponent, dotted curve for a power-law fit with the exponent fixed at 0.6 and dashed for a linear fit. The light grey solid curve outlines the CaII K intensity binned in 70 intervals containing an equal number of points. Histograms on the left show the distribution of magnetic field strength in 4 subranges of the CaII K-line flux. Histograms on top of the central panel depict the distribution of calcium intensity in 4 subranges of the magnetic flux. For each histogram the corresponding range of considered values is marked in the main figure: by horizontal solid lines for magnetic flux distributions and by vertical dotted lines for the CaII K-line flux histograms. The number of counts, $n$, represented in the histograms are indicated.

well as for the relations between UV intensity at $1600 \AA$ and magnetic flux, and between $3.5 \mathrm{~mm}$ brightness and magnetic flux. Regarding the dependence of millimeter brightness on magnetic field strength (not shown), the data also strongly suggests that it is nonlinear and among the considered fitting functions is best described by a power law, but the sizeable scatter does not allow the exponents of the fit to be determined reliably.

Next we employed a brightness threshold technique for calcium intensity image and created a binary map discriminating between the areas representing cell interiors or internetwork (IN) and chromospheric network (NW). We studied the relations between the chromospheric emission and the magnetic flux separately for IN and NW locations. On the whole, chromospheric emission displays a rather different dependence on the network and internetwork magnetic field (see Fig. 3 for calcium intensity). For the network, again as in the case of the global FOV, the relations between all three chromospheric quantities and magnetic field are best described by a power-law. In Table 2 we list the exponents from the best-fit power laws for the images at the resolution of the MDI magnetograms ( 4 arcsec) and of the BIMA images $(12$ arcsec). The exponents of the relation for calcium intensity are found to be similar to $0.5-0,6$, cited by Schrijver et al. (1989) for the active region and by Harvey \& White (1999) for the quiet-Sun network. 

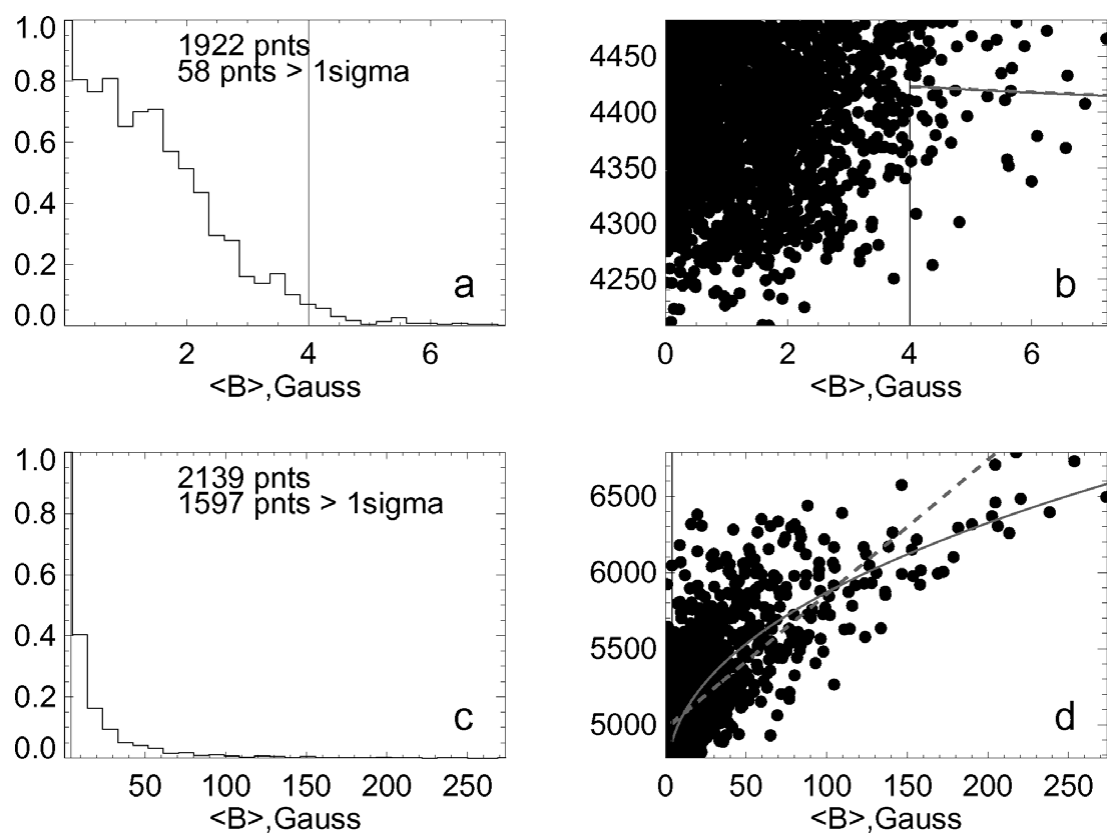

Figure 3. Comparison of IN and NW emission averaged over $0.5 \mathrm{~h}$ : histograms of a) IN and c) NW magnetogram signal, $\mathbf{b}, \mathbf{d}$ ) CaII K-line flux vs. absolute value of the IN and NW magnetic field, respectively. The solid vertical lines represent $1 \sigma$-noise of the magnetogram signal.

Table 2. Dependence of the network emission on the magnetic flux: exponents of the power-law fits calculated for the images at 4 arcsec and 12 arcsec resolution, averaged over $3.5 \mathrm{~h}$ and $0.5 \mathrm{~h}$. In the last column we list for comparison the exponents of the power-law fits calculated globally in the field of view.

\begin{tabular}{|c|c|c|c|c|c|}
\hline \multirow[t]{2}{*}{ NW flux } & \multicolumn{2}{|c|}{$4 \operatorname{arcsec}$ resolution } & \multicolumn{2}{|c|}{$12 \operatorname{arcsec}$ resolution } & \multirow[t]{2}{*}{ FOV flux } \\
\hline & $3.5 \mathrm{~h}$ & $0.5 \mathrm{~h}$ & $3.5 \mathrm{~h}$ & $0.5 \mathrm{~h}$ & \\
\hline$|B|-I_{\text {CaIIK }}$ & 0.6 & 0.43 & 0.54 & 0.47 & 0.31 \\
\hline$|B|-I_{1600 A}$ & 0.49 & 0.53 & 0.66 & 0.44 & 0.36 \\
\hline
\end{tabular}

In the internetwork regions almost no dependence of the calcium, UV at $1600 \AA$ and $3.5 \mathrm{~mm}$ intensity on the magnetogram signal was found. However, due to the very low $B$ values in the internetwork, as seen in Fig. 3, top panel, only a few percent of IN structures are associated with magnetogram signal exceeding noise, and this makes a confident study difficult. Nonetheless, our result confirms the conclusions drawn in the recent paper by Rezaei et al. (2007), and supports the idea that bright locations in the internetwork are due to nonmagnetic heating (e.g., acoustic heating).

\section{Summary and Conclusions}

(a) Summarizing, clear spatial correlations found between photospheric magnetograms, calcium K-line, UV intensity at $1600 \AA$ and $3.5 \mathrm{~mm}$ emission indicate that heating in the quiet-Sun lower and middle chromosphere maps out the underlying photospheric magnetic field rather well. This implies that on the chromospheric heights covered by the analyzed here wavelengths we deal with the same heating mechanism. 
(b) However, different chromospheric brightness structures, such as supergranular cell boundaries and cell interiors, are probably due to different heating processes (magnetic and nonmagnetic).

(c) In general, present data on both chromospheric emission and magnetic field are not sufficient to establish physical mechanisms that are acting at chromospheric heights leading to the observed brightness structures.

(d) In this respect, higher sensitivity magnetograms are needed, and regarding the chromospheric data an extraordinarily powerful tool to study the thermal structure of the chromosphere will be provided by Atacama Large Millimeter Array (ALMA, Bastian 2002; Loukitcheva et al. 2008).

\section{Acknowledgements}

Millimeter-wavelength astronomy at the University of Maryland is supported by NSF grant AST-0540450. Solar research at the University of Maryland is supported by NASA grants NNX06-AC18G and NNX08-AQ48G. M. Loukitcheva acknowledges support from MK-1853.2007.2 of the Grant Council of the President of the Russian Federation.

\section{References}

Bastian, T. S. 2002, AN 323, 271

Handy, B. N. \& others 1999, Sol.Phys. 187, 229

Harvey, K. \& White, O. 1999, ApJ 515, 812

Leighton, R. B. 1959, ApJ 130, 366

Loukitcheva, M., Solanki, S. K., \& White, S. M. 2008, Ap\&SS 313, 197

Loukitcheva, M., Solanki, S. K., \& White, S. M. 2009, A\&A, in press

Nindos, A. \& Zirin, H. 1998, Solar Phys. 179, 253

Rezaei, R., Schlichenmaier, R., Beck, C. A. R., Bruls, J., \& Schmidt, W. 2007, A\& A 466, 1131

Rutten, R. J. \& Uitenbroek, H. 1991, Solar Phys. 134, 15

Scherrer, P. H. \& others 1995, Solar Phys. 162, 129

Schrijver, C. J. 1987, A\&A 172, 111

Schrijver, C. J. 1992, A\&A 258, 507

Schrijver, C. J., Cotè, J., Zwaan, C., \& Saar, S.H. 1989, ApJ 337, 964

Sivaraman, K. R. \& Livingston, W. C., 1982, Solar Phys. 80, 227

Skumanich, A., Smythe, C., \& Frazier, E. N. 1975, ApJ 200, 747

\section{Discussion}

Kouтснму: Two comments. 1) Intranetwork magnetic elements are small bi-poles (both polarities are present) which emerge and migrate towards the boundary of the cell. 2) Network elements are rather uni-polar magnetic elements collected at the vertex of converging flows due to super granulation. I guess this makes the difference.

LOUKITCHEVA: Yes, intranetwork \& network elements are of different nature. We are discriminating between these two and study their emission and dependence on magnetic flux separately. And we obtain different relationships for magnetic elements and chromospheric emission. To take into account the dynamic behavior of intra-network elements, their proper motions, we studied them from very short time averages. And for network elements, which are more stable in time, longer time integration was applied. 